\title{
Rapidly prototyping biocompatible surfaces with designed wetting properties via photolithography and plasma polymerization
}

\author{
M. F. Berwind ${ }^{1,2}$ - A. Hashibon ${ }^{1}$ A. Fromm ${ }^{1}$ M. Gurr ${ }^{1}$ F. Burmeister ${ }^{1}$ C. Eberl ${ }^{1,2}$
}

Received: 22 February 2017 / Accepted: 9 August 2017 / Published online: 17 August 2017

(C) The Author(s) 2017. This article is an open access publication

\begin{abstract}
The testing and manufacturing of lab-on-a-chip devices increasingly require lower lead times from conception to market. In order to supply a potential answer to this burgeoning demand, the work herein explores a design and prototyping process for biocompatible surfaces with designed wetting states ranging from slightly hydrophilic behavior to superhydrophobicity. Direct laser writing was used to fabricate geometrically parameterized base structures out of an acrylic photopolymer (IP-Dip) for eventual coating of samples with hexamethyldisiloxane (HMDSO) via plasma polymerization, with numerical methods validating candidate geometries. After manufacturing, samples were examined for structural and/or coating integrity via scanning electron microscopy and optical microscopy; subsequent wetting property evaluation was performed with a contact angle goniometer. Beginning with the slight hydrophilicity of the planar-coated base polymer, surfaces were shown displaying wetting behavior from the 'mushroomed' Wenzel state, the metastable Cassie-Baxter state, and superhydrophobicity with the non-wetting rolling predicted by literature. The combination of methods used in this work creates a parameter space for the rapid fabrication
\end{abstract}

Electronic supplementary material The online version of this article (doi:10.1007/s10404-017-1984-6) contains supplementary material, which is available to authorized users.

M. F. Berwind

matthew.berwind@iwm.fraunhofer.de

1 Materials Design Department, Fraunhofer Institute for Mechanics of Materials (IWM), 79108 Freiburg, Germany

2 Institute for Microsystems Engineering, Chair for Micro and Materials Mechanics, Albert-Ludwigs University of Freiburg, 79110 Freiburg, Germany of 'designer' or 'programmable' surfaces, that is, the attainment of a specific wetting state through parametric variation with fast prototype processing times on the order of hours or days instead of weeks or months, and is typified by a presented microarray section. Of further specific relevance to the lab-on-a-chip community is the biocompatible nature of the HMDSO coatings. All structures presented in this work can be used as printed on any substrate or transferred for further processing into media more applicable for largescale manufacturing.

Keywords Microfluidics · Lab-on-a-chip ·

Stereolithography $\cdot$ Plasma polymerization $\cdot$ Materials design

\section{Introduction}

Changing the microstructuring of a surface, and thus the resultant surface curvature of a liquid resting on it, theoretically (Dorrer and Rühe 2009) allows an energy balance with a perfectly non-wetting, superhydrophobic state. Much work has been invested in the fabrication of surfaces with superhydrophobic behavior (Celia et al. 2013) with the design of specific wetting states now becoming an area of interest (Fernández et al. 2017). Advancements in the processing speed and accuracy of laser lithography have vastly increased the ease of creating finely structured surfaces with designed wetting properties on numerous substrates with myriad application areas (Ueda and Levkin 2013). One of these fabrication methods, direct laser writing (DLW), currently shows particular promise for the fabrication of prototypes containing simple repetitive to highly complex 3D structures with submicron feature sizes across square millimeters and centimeters, readily 


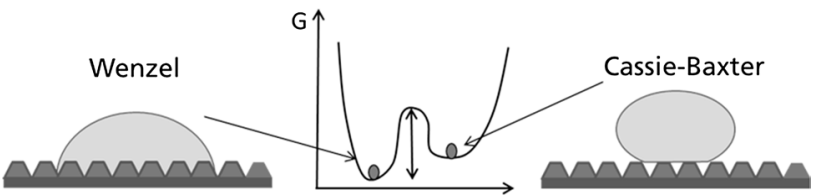

Fig. 1 Wenzel and Cassie-Baxter wetting with their corresponding energy states

answering the fabrication questions required for the careful design of a surface's microstructure. A parametric approach to the surface design prototyping supported by contact angle calculations can feasibly result in desired custom wetting parameters with low cost. When initially evaluating these wetting characteristics, two wetting models for rough surfaces are useful to frame discussion in this paper: the Wenzel and Cassie-Baxter models (Wenzel 1936). Wenzel's model takes a homogeneous wetting regime, while the Cassie-Baxter model is for a heterogeneous state, as illustrated in Fig. 1. There are transition states between these two common models, namely ones that display different degrees of heterogeneity or 'mushrooming' from structure tips to substrate. The basic underlying physics consists of comparing the energies of a configuration in which the droplet is completely wetting the surface (Wenzel state in Fig. 1) and that in which the drop is metastably resting on top of the patterns (Cassie-Baxter state in Fig. 1). Derivations can be found in classical texts (Wenzel 1936) and in Whyman et al. 2008). The condition for a metastable Cassie-Baxter state is that the contact angle of the patterned surface is larger than a critical wetting angle $\boldsymbol{\theta}_{\mathbf{c}}$ given by:

$\cos \boldsymbol{\theta}_{\mathbf{c}} \geq+\frac{\phi_{\mathrm{s}}-1}{\boldsymbol{r}-\phi_{\mathrm{s}}}$

However, this is normally insufficient since in practical systems, other factors need to be considered. The above theoretical treatment assumes a rotationally invariant system and excludes both impurities and the intricate shape of the triple line and the complex shape of the solid-air-liquid interface beneath the drop. Furthermore, the practically important impact of dynamic wettability (Oener and Mccarthy 2000) is not included. Such factors are not amenable to simple analytical solutions and therefore need to be calculated numerically. Several methods, including computational fluid dynamics (CFD) and multi-scale modeling (Giro et al. 2014; Karapetsas et al. 2017), allow the achievement of a robust design for a sufficiently stable Cassie-Baxter state. This design approach enables a parametrization of the particular geometry giving specific values of $\phi_{\mathrm{s}}$ in Eq. 1 above and therefore values of $\boldsymbol{\theta}_{\mathbf{c}}$. The parameterization was then further refined based on computational modeling resulting in a set of candidate surfaces for specific wetting behaviors (Hashibon et al. 2014). Photolithography is a known method for creating superhydrophobic surfaces (Kawai and Nagata 1994), but the industrial relevance of such a parameterized approach to prototyping has been greatly enhanced by the speed and ease of fabricating samples with submicron dimensions via direct laser writing. The result is a scientist or engineer receiving concrete results closer to her application cheaper and faster than before. This manuscript demonstrates the validation of this manufacturing and design strategy, with direct laser writing's adaptability and processing speed with substrate coverage areas in the square millimeter range being ideal for the requirements of such a parametric study. Plasma polymerization of HMDSO and other organosilicons in and of itself has been reported before (Wagterveld et al. 2006; Tsuruta et al. 2006) to achieve superhydrophobic wetting conditions, but is used in this work as a method of increasing the parametric design space while simultaneously potentially opening application areas in which biocompatibility (Noorisafa et al. 2016; Bo et al. 2016; Ciasca et al. 2016; Falde et al. 2016) and larger substrate sizes are of importance.

\section{Experimental}

\subsection{Lithographic fabrication: DLW}

Structures to be fabricated on the substrate surface are first modeled using CAD (Fig. 2). The CAD file is then converted to a standard lithography file, which can then be translated into coordinates to be polymerized via photon absorption. These coordinates contain stepwise bottomup path traces representing design geometries and laser power information. The coordinates and laser intensities are then compiled and translated into commands for commercially available DLW equipment (Nanoscribe $\mathrm{GmbH}$ ). A vast array of substrate materials is available to the user. For the purposes of these experiments, $<100>$ oriented silicon wafers with a $2.5 \mathrm{~cm}$ diameter were chosen due
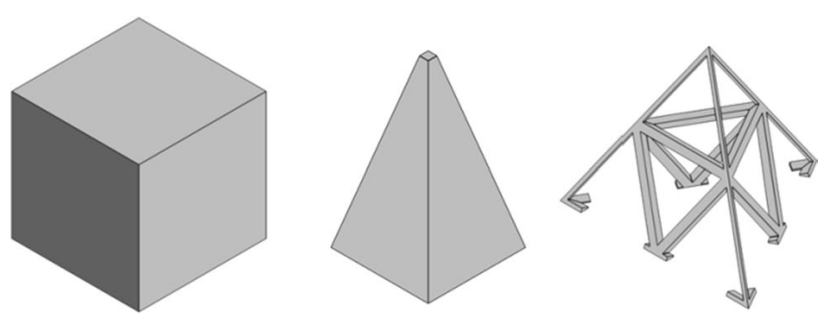

Fig. 2 Shapes lithographically patterned in this study. From left to right, cube, truncated pyramid, and strut tetrahedron. Planar structures were also studied for a base reference 
to compatibilities with lithography and further handling (chemical cleaning and plasma polymerization). Structures are then patterned on substrates either in simple squares or in alternating truncated pyramid-strut tetrahedron bands designed to mimic fluid separation microarrays.

The UV hardening photoresist, IP-Dip (Nanoscribe $\mathrm{GmbH}$ ), is then drop cast onto the silicon wafer. Pivoted galvo-mirrors are responsible for scanning the focal point of a femtosecond laser in the exposed photoresist volume. This additive fabrication technique leaves a form true hardened polymer structure after chemical cleaning. The lithographic surface structuring involved in this study typically lasts only several (less than 1-6) hours, depending mostly on the volume to be fabricated.

\subsection{Plasma polymerization}

After nanolithographic fabrication of all geometric types, charges from each sample type with an additional, partially masked silicon substrate were transferred to a S400-S3 coating system by FHR, Dresden, Germany (FHR Anlagebau $\mathrm{GmbH})$. The pressure in the recipient was lowered to $2 \mathrm{E}-7$ mbar. For the deposition, HMDSO was vaporized in a self-built vaporizer at room temperature and led into the recipient as a precursor. The RF power during the deposition was set to $100 \mathrm{~W}$ for $90 \mathrm{~s}$ with a constant frequency of $13.56 \mathrm{MHz}$ and a constant deposition pressure of $3 \mathrm{E}-2$ mbar. The coating thickness of $10 \mathrm{~nm}( \pm 2)$ was determined by measuring the resulting step height of the edge on the partially masked silicon substrate with white light interferometry using the VSI mode of a WYKO NT1100 by Veeco Inc., NY, USA (Veeco Inc.).

\subsection{Goniometric testing}

Goniometric experiments were performed to measure the static and dynamic sessile, advancing, and receding contact angles of water on the various structured IP-Dip surfaces, with and without HMDSO coating. For static sessile tests, a $1 \mu \mathrm{L}$ droplet of deionized water was lowered to the surface until a snap-on event occurred. In the event that no snap-on event occurred with the starting volume, the volume of the water was increased until a drop could be removed from the goniometer's needle head, which was the case with the increasingly hydrophobic samples. The volume changing method was used for dynamic sessile tests. All contact angles resultant from these two methodologies were extracted from experimental images using a high accuracy B-spline method (Stalder et al. 2006), with results summarized in Tables 1 and 2, respectively. Furthermore, water droplets were transported on the alternating patterned surfaces mimicking droplet separation microarrays to test local wetting properties. Droplets, still attached to the goniometer's dispenser needle, were moved along the substrate via translation of the goniometer stage resulting in dynamic test conditions with droplet front advancement orthogonal to wetting tips.

\section{Results and discussion}

The first structure is a simple planar IP-Dip coating. Lithographic fabrication of this simple plane is preferred to other facile methods such as spin coating, as the 'hatching' writing strategy executed during DLW certainly affects wetting conditions. A second substrate coating routine creates a matrix of cubes separated by a given distance;

Table 2 Dynamic sessile advancing and receding contact angles as measured by the volume changing method

\begin{tabular}{lrlll}
\hline & \multicolumn{1}{c}{$\theta_{\text {adv }}$} & $\theta_{\text {rec }}$ & $\theta_{\text {adv }}($ HMDSO $)$ & $\theta_{\text {rec }}($ HMDSO $)$ \\
\hline Flat & $79.9^{\circ}$ & $59.5^{\circ}$ & $100.1^{\circ}$ & $82.9^{\circ}$ \\
Cubic & $87.7^{\circ}$ & $35.7^{\circ}$ & $150.2^{\circ}$ & $94.0^{\circ}$ \\
Pyramidal & $101.1^{\circ}$ & $34.1^{\circ}$ & $128.2^{\circ}$ & $69.9^{\circ}$ \\
Tetrahedral & $63.1^{\circ}$ & $64.7^{\circ}$ & - & - \\
\hline
\end{tabular}

Table 1 Goniometric sessile contact angle measurements on geometrically parameterized uncoated IP-Dip and HMDSOcoated IP-Dip wetting surfaces

\begin{tabular}{|c|c|c|c|c|c|c|}
\hline & & & ${ }_{\mathrm{b}}^{\mathrm{a}}$ & & & \\
\hline & $a(\mu \mathrm{m})$ & $b(\mu \mathrm{m})$ & $h(\mu \mathrm{m})$ & $s(\mu \mathrm{m})$ & IP-Dip, $\theta_{\mathrm{c}}$ & $\mathrm{HMDSO}, \theta_{\mathrm{c}}$ \\
\hline Flat & 100 & 100 & 1.5 & 0 & $80.5^{\circ}$ & $106.4^{\circ}$ \\
\hline Cubic & 110 & 110 & 110 & 5 & $91.9^{\circ}$ & $132.4^{\circ}$ \\
\hline Pyramidal & 10 & 110 & 187 & 5 & $108.5^{\circ}$ & $173.9^{\circ}$ \\
\hline Tetrahedral & 0.45 & 95 & 110 & 10 & $130.2^{\circ}$ & - \\
\hline
\end{tabular}


the height of the cubes ensures that the substrate initially plays no role in the wetting state. The third structure introduces tips to form a truncated pyramid. The truncated pyramid, with its square tip measuring $10 \mu \mathrm{m}$ a side and its gap spacing of $5 \mu \mathrm{m}$, and resultant tip-to-tip spacing of $115 \mu \mathrm{m}$, represents a structure that could feasibly be created via traditional micromachining techniques. A fourth structure, a braced or strut tetrahedral concept, was designed to maintain structural stiffness and limit lithographically written material volume while simultaneously utilizing the tip-writing resolution capabilities provided by direct laser writing. Such structures with a high stiffnessto-weight ratio are also interesting in situations requiring mechanical robustness, which drastically affects wetting behavior in applications (Kondrashov and Rühe 2014; Tian et al. 2016), even more so if the full volumes were written. All four different surface geometry types were printed in matrices to cover an area of $2.5 \times 2.5 \mathrm{~mm}^{2}$. The four different structures were chosen to parametrically explore important variables in wetting design: covered substrate area, structure height from substrate, structure spacing, and tip sharpness; their respective contact angle measurements listed in Table 1 represent the average of the left and right contact angles of a static sessile water droplet. The planar IP-Dip surfaces showed mildly hydrophilic wetting behavior, while its HMDSO-coated counterpart was mildly hydrophobic. These two planar structures acted as a base reference for measurements performed on the subsequent samples. The structure types were each chosen to display a specific tailored wetting state, a capability of critical importance to cost-effective industrial prototyping.

Although all HMDSO-coated structures showed hydrophobicity, the largest measureable static sessile contact angle of $173.9^{\circ}$ was found with the truncated pyramids and is shown in Fig. 4-d. The techniques employed were capable not only of achieving contact angles very close to intended value ranges, but they were also capable of changing from specific wetting states. It should be noted that the sessile contact angle in Table 1 of the tetrahedral brace structures could not be measured due to the superhydrophobic behavior. Due to the heights and the tip-to-tip spacing of the pyramidal and braced tetrahedral samples, wetting or 'Cassie mushrooming' of the underlying substrate could be achieved during sessile wetting of these sample types. This suggests that such structures, although feasible in terms of machining, are not perfectly non-wetting without further treatment when made of IP-Dip. An HMDSO coating of the truncated pyramids showed highly hydrophobic wetting behavior, but still did not result in perfect non-wetting, showing a Cassie-Baxter state with mushrooming. Superhydrophobic behavior was repeatedly achieved with the brace tetrahedral structures after HMDSO coating. Using this wetting angle goniometer, it was not possible, regardless of droplet volume, to place a sessile droplet on the HMDSOcoated braced tetrahedron lattice, or to achieve measureable mushrooming. For this reason, a measured contact angle is absent from Table 1. Experimental videos show a 'rolling' mechanism (Fig. 3) of motion predicted by Richard and Quéré (1999). By parametrically varying structural characteristics that impact surface roughness in wetting, this work was able to achieve designed wetting states with a rational design approach. During dynamic contact angle measurement of the pyramidal structures, the mushroomed wetting state of the droplet is maintained throughout advancing and receding steps. This is true for both HMDSO-coated samples and uncoated samples and is in stark contrast to the tetrahedral structures, which show greatly reduced advancing and receding contact angles in comparison with sessile tests (see Table 2). It is suspected that the porous nature of the braced tetrahedron structures results in both the repeatedly lower than expected advancing and receding contact angles as well as the very low hysteresis. A possible explanation is the subsurface domination of wetting behavior after forced fluid advancement due to penetration allowed by air gaps, a complicated triple line energy barrier that, once surpassed, results in high wetting and 'snap-on' to the subsurface. This suggests that the braced tetrahedron structures are not suitable in some dynamic situations without a silane coating - an important insight regarding the effect of porosity on applications. The key upshot of this study, as presented in Fig. 4, illustrates this 'wetting by design' approach and a sample set of wetting states capable of being attained by such a strategy. These capabilities lend themselves readily toward microarray fabrication and/or high-throughput screening applications due to their ability to realize specific sections of a substrate with known and alternating wetting profiles. Substrates were fabricated with this technique typifying the fundamental concepts of fluid transport/separation microarrays (Fig. 5) and consist of a braced tetrahedral region, the superhydrophobic section, along with a truncated pyramid region, a region where mushrooming is allowed. The aim of these regions is to form a spatial separation for the formation of small-volume droplets, essentially two areas with homogeneous (Wenzel) and heterogeneous (Cassie-Baxter) wetting regimes. Droplet transportation orthogonal to these regions showed distinct 'pass' and 'no-pass' regions (see supplemental material) that can be tuned to specific application requirements.

\section{Conclusions}

DLW in combination with plasma polymerization enables the rapid parameterized prototyping of highly structured (submicron feature size) surfaces with a wide range of designed wetting states. Parametrically varying geometric 
Fig. 3 a $\times 5$ micrograph of lithographically fabricated tetrahedral structures. $\mathbf{b} \times 20$ magnification micrograph of the same patterned substrate. c Superhydrophobic 'rolling' droplet behavior on the patterned substrate after HMDSO plasma polymerization

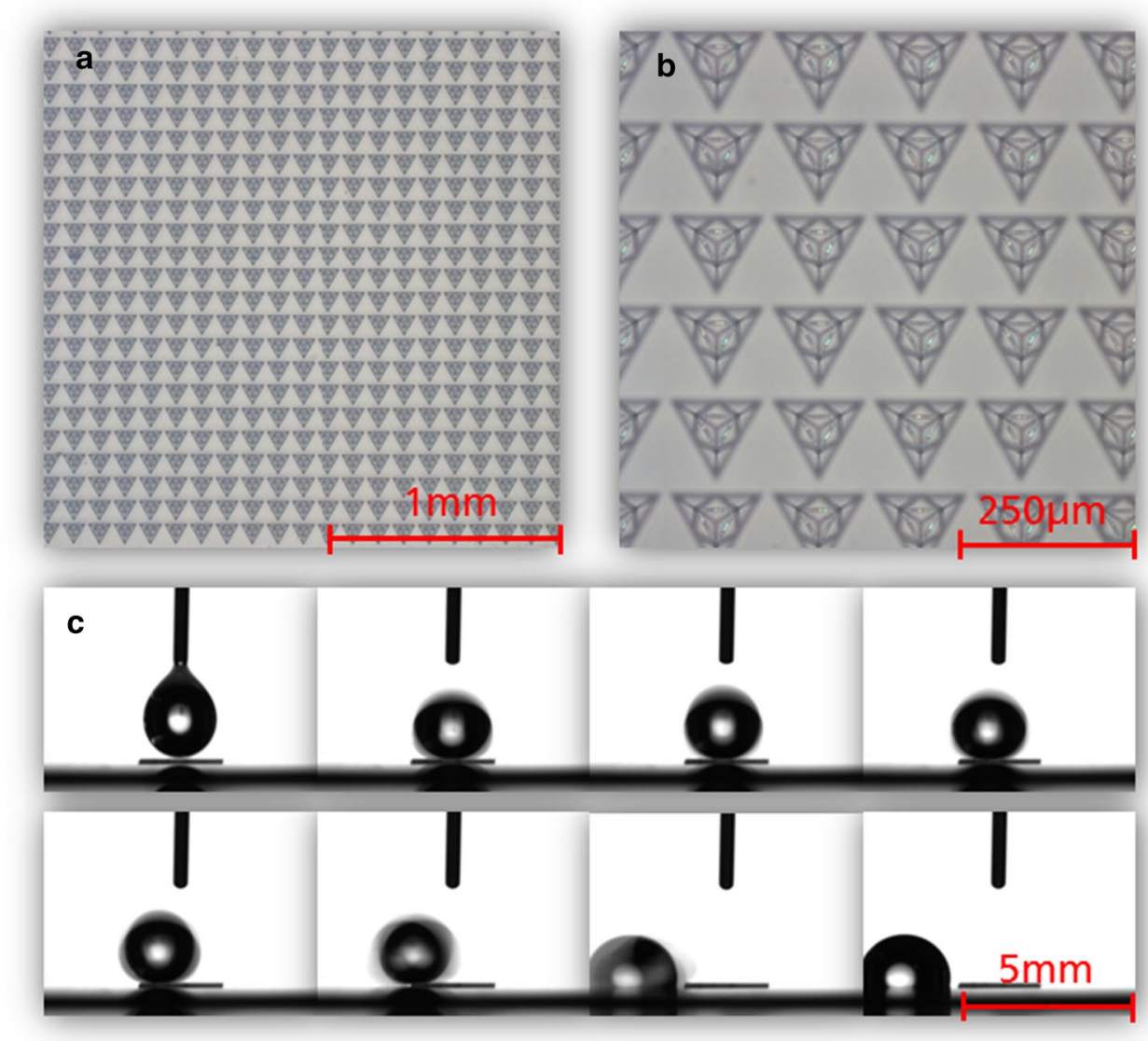

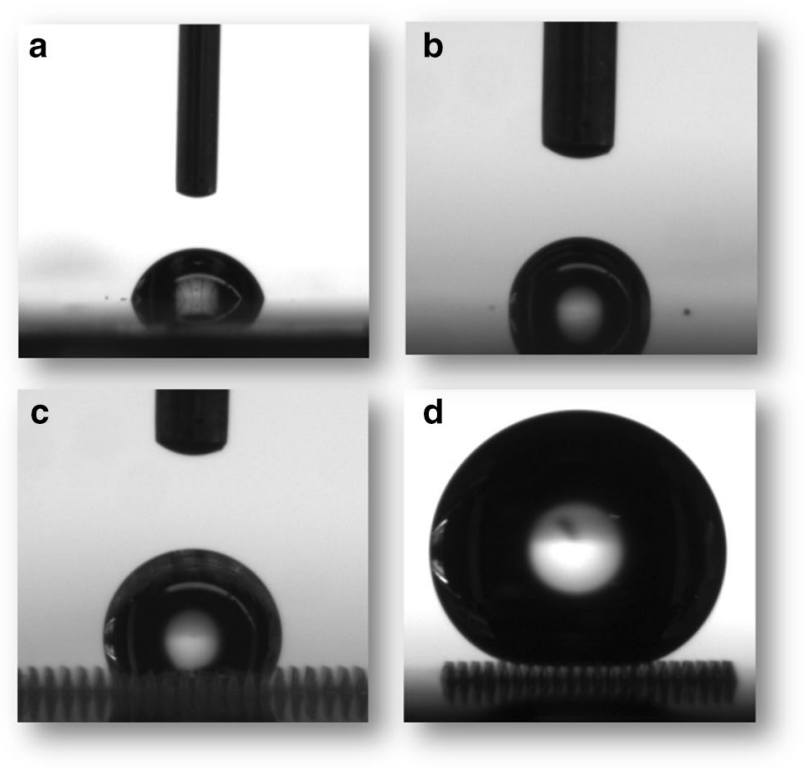

Fig. 4 a Hydrophilic base substrate. b Hydrophobic HMDSO-coated substrate. c Truncated pyramids showing Wenzel state. d HMDSOcoated truncated pyramids showing the metastable Cassie-Baxter state components of a surface allows a rational and applicationspecific design approach toward the control of interface energy and wetting state. Furthermore, patterned surfaces including wetting state gradients could be employed in situations in which hydrophobicity/hydrophilicity, namely surface tension confined microfluidics, is the driving force of mass transport. Simple two-dimensional patterns, however, are not the only type of structures capable of being fabricated with this approach; the computation and experimental techniques used in this work can also be used for the fabrication of multilayer or more three-dimensionally complex systems. Although the strut tetrahedron structures showed the desired superhydrophobicity along with short lithographic processing times, these structures are likely not feasible should upscaling to different manufacturing techniques be desired. In that case, such strut structures should be replaced by more mechanically robust whole volumes. This would result in further mechanical robustness as well as enhanced hydrophobicity in dynamic wetting scenarios due to the decreased structural porosity discussed earlier. Such whole volume structures, akin to the truncated pyramids, could then be feasibly fabricated with micromachining and manufacturing techniques and then used for further upscaling. A proof of concept for a 'structure to wetting state' design approach 
Fig. 5 Schematic view of structured surface showing varying degrees of hydrophobicity/ hydrophilicity, the foundation for droplet separation arrays

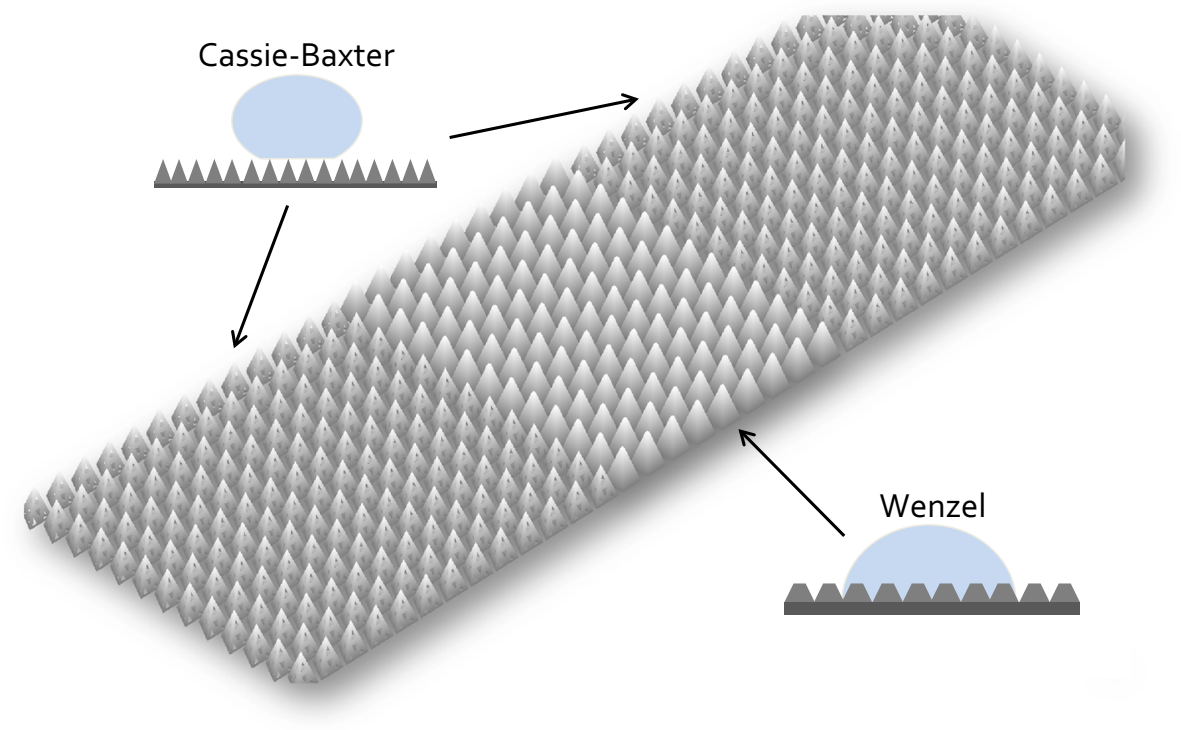

is presented in this paper and is illustrated by goniometric tests performed on fabricated samples, with results ranging from a slightly hydrophilic contact to superhydrophobicity. Such a design approach could be used to directly fabricate patterned surfaces with desired wetting behavior for a fluid transport system, as was done in this work, or they could also be used to create a negative replica for further fabrication processing. To the authors' knowledge, this is a new finding in the microfluidics community, with previous work having only achieved wetting state control by varying the droplet impact velocity (Fernández et al. 2017). Combining this novel design capability with plasma polymerization, for instance of HMDSO, opens further application possibilities in biocompatible systems for liquid mass transport for chemical and medical analyses. Plasma polymerization is essentially another degree of freedom in the design process, capable of increasing hydrophobicity of a base material limited by its specific surface chemistry while also possessing high scalability. In addition to these benefits, using coating via plasma polymerization enables substrate materials of any kind to match from the prototyping stage to the upscaled industrial stage since the coating dominates the surface wetting characteristics. The structuring freedom and speed at which the utilized methods allow sample fabrication are of particular relevance to research and industry; any application tailored wetting state can be achieved within hours instead of months, decreasing lead time from development to implementation. Robust prototyping capabilities with a wide range of applications are made possible through the combination of design, geometric structuring freedom provided by DLW, and the scalability of plasma polymerization.

Open Access This article is distributed under the terms of the Creative Commons Attribution 4.0 International License (http:// creativecommons.org/licenses/by/4.0/), which permits unrestricted use, distribution, and reproduction in any medium, provided you give appropriate credit to the original author(s) and the source, provide a link to the Creative Commons license, and indicate if changes were made.

\section{References}

Bo F, Giner I, Keller A, Grundmeier G, Fischer H (2016) Plasmaenhanced chemical vapor deposition (PE-CVD) yields better hydrolytical stability of biocompatible SiOx thin films on implant alumina ceramics compared to rapid thermal evaporation physical vapor deposition (PVD). ACS Appl Mater Interfaces 8:1780517816. doi:10.1021/acsami.6b04421

Celia E, Darmanin T, De Givenchy ET, Amigoni S, Guittard F (2013) Recent advances in designing superhydrophobic surfaces. J Colloid Interface Sci 402:1-18. doi:10.1016/j.jcis.2013.03.041

Ciasca G, Papi M, Businaro L, Campi G, Ortolani M, Palmieri V, Cedola A et al (2016) Recent advances in superhydrophobic surfaces and their relevance to biology and medicine. Bioinspir Biomim 11(1):1-15. doi:10.1088/1748-3190/11/1/011001

Dorrer C, Rühe J (2009) Some thoughts on superhydrophobic wetting. Soft Matter 5(1):51-61. doi:10.1039/b811945g

Falde EJ, Yohe ST, Colson YL, Grinstaff MW (2016) Superhydrophobic materials for biomedical applications. Biomaterials 104:87103. doi:10.1016/j.biomaterials.2016.06.050

Fernández A, Francone A, Thamdrup LH, Johansson A, Bilenberg B, Nielsen T, Guttmann M, Sotomayor Torres CM, Kehagias N (2017) Design of hierarchical surfaces for tuning wetting characteristics. ACS Appl Mater Interfaces 9:7701-7709

"FHR Anlagebau GmbH." n.d. Am Hügel 2, 01458 Ottendorf-Okrilla

Giro R, Bryant PW, Steiner MB, Del Grande RR, Feger C, Engel M (2014) Multi-scale modeling of wetting : effects of surface roughness. In: Iberian Latin American congress on computational methods in engineering

Hashibon A, Gurr M, Polfer P, Burmeister F, Kraft T (2014) Computational design of wetting and spreading behavior for enhanced in-vitro-diagnostic applications. In: Proceedings of the 2nd international conference on micro fluidic handling systems

Karapetsas G, Lampropoulos NK, Dimakopoulos Y, Tsamopoulos J (2017) Transient flow of gravity-driven viscous films over 
3D patterned substrates: conditions leading to Wenzel, Cassie and intermediate states. Microfluid Nanofluid 21(17):1-20. doi:10.1007/s10404-017-1853-3

Kawai A, Nagata H (1994) Wetting behavior of liquid on geometrical rough surface formed by photolithography. Jpn J Appl Phys 33:1283-1285

Kondrashov V, Rühe J (2014) Microcones and nanograss: toward mechanically robust superhydrophobic surfaces. Langmuir 30:4342-4350

"Nanoscribe GmbH." n.d. 1, Hermann-von-Helmholtz-Platz Eggenstein-Leopoldshafen, 76344 Deutschland

Noorisafa F, Razmjou A, Emami N, Low Z-X, Korayem H, Kajani AA (2016) Surface modification of polyurethane via creating a biocompatible Superhydrophilic nanostructured layer: role of surface chemistry and structure. J Exp Nanosci. doi:10.1080/17458080. 2016.1188223

Oener D, Mccarthy TJ (2000) Ultrahydrophobic surfaces. Effects of topography length scales on wettability. Langmuir 16(20):7777-7782

Richard D, Quéré D (1999) Viscous drops rolling on a tilted non-wettable solid. Europhys Lett 48(3):286-291

Stalder AF, Kulik G, Sage D, Barbieri L, Hoffmann P (2006) A snakebased approach to accurate determination of both contact points and contact angles. Colloids Surf A Physicochem Eng Asp 286(1-3):92-103

Tian X, Verho T, Ras RHA (2016) Moving superhydrophobic surfaces toward real-world applications. Science 352(6282):142-143

Tsuruta S, Morimoto K, Hirotsu T, Suzuki H (2006) Superhydrophobic phenomena on three-dimensional surface structures coated with plasma polymer. Jpn J Appl Phys 45(10B):8502-8505. doi:10.1143/JJAP.45.8502

Ueda E, Levkin PA (2013) Emerging applications of superhydrophilicsuperhydrophobic micropatterns. Adv Mater 25(9):1234-1247. doi:10.1002/adma.201204120

"Veeco Inc." n.d. 1 Terminal Dr, Plainview, NY 11803, USA

Wagterveld RM, Berendsen CWJ, Bouaidat S, Jonsmann J (2006) Ultralow hysteresis superhydrophobic surfaces by excimer laser modification of SU-8. Langmuir 22(26):10904-10908

Wenzel R (1936) Resistance of solid surfaces to wetting by water. Ind Eng Chem 28(8):988-994

Whyman G, Bormashenko E, Stein T (2008) The rigorous derivation of Young, Cassie-Baxter and Wenzel equations and the analysis of the contact angle hysteresis phenomenon. Chem Phys Lett 450:355-359. doi:10.1016/j.cplett.2007.11.033 